\title{
Behavior of Recycled Asphalt to the Fatigue
}

\author{
Bordjiba $\mathrm{A}^{1 *}$, Guenfoud $\mathrm{H}^{2}$
}

Department of Architecture, Badji Moukhtar University, Annaba, Algeria Department of Civil Engineering, 08 may 1945, Guelma, Algeria

Corresponding Author: bordjibaabdelhak@gmail.com

Received: 23-03-2018

Accepted: 22-01-2019

\begin{abstract}
Climatic and traffic effects are the causes of aging of the surface layer of asphalt, which leads to the necessary renovation of the latter. The use of waste from the tread layer provides a viable and beneficial solution for the environment and the economy. However, this solution must meet the requirement of sustainable development, which necessitates that a road has a very long lifespan. In this study we investigated the performance of recycled asphalt in terms of fatigue (as an element of life-cycle control). All the formulas were tested by the fatigue test in order to define the influence of the proportions of the asphalt aggregates, penetrability of the new binder used, and the manufacturing temperature. The results obtained showed the relationship between the contribution binder (reproduced between the new binder and the old binder) and the fatigue resistance under the influence of the parameters that we have mentioned above. At the end an optimization study has been carried in order to determine exactly the doses required to formulate recycled asphalt resisted to fatigue, the optimization seeks to maximize the asphalt aggregates and minimized the temperature of manufacture with a class of the binder compatible.
\end{abstract}

Key words: Asphalt, fatigue, Binder, Environment, Penetrability.

\section{Introduction}

Surfaced roads age under the effects of traffic and climate, which lead to the end of their life, in increments that reflect the functions required by the regulations, or the needs envisaged by the employer, (Abdelhak et al., 2016-a). In Algeria, the asphalt aggregates for the maintenance of the rolling asphalt layer are recycled into nature or reused as a foundation layer for low-traffic roads, (Pascal, 2017). However, after the Rio Summit in 1992, environmental awareness has emerged that sustainable development is the link between economic growth and environmental protection (Kalantar et al., 2012). On applying the concept of sustainable development in the field of road construction, the latter requires minimizing the use of non-renewable natural raw material and the construction of a road surface that has a very long lifespan, (Technical Guides and General Studies- CTTP, 2015). The logical answer to this problem is recycling, which imposes the valorization of the deposit of materials already existing in the surfacing, and limits the amount of new materials in the formulation of the new asphalt, (Haddad et al., 2017). In this study we checked the effect of the penetrability of the contribution binder, asphalt aggregate dosing and the temperature of manufacturing on the resistance to fatigue of recycled asphalt. The formulated type of asphalt is BBSG 0/14 (semi-dense bituminous concrete), because this is the type most commonly encountered on Algerian roads. The hypothesis in this work is that asphalt aggregates have a percentage of old binder which can cause fatigue for recycled asphalt, but that very precise control of the 'contribution binder' (old binder + new binder) of recycled asphalt can show us a new binder corresponding to the percentage of asphalt aggregates used. Such close control also permits analysis of the temperature of manufacturing, such that the mixture (old binder and new binder) may take the form of a contribution binder compatible with the features of recycled manufactured asphalt. The asphalts were subjected to stresses of short durations, repeated over time. These represented the successive passages of the axles of 
vehicles, (Kavussi \& Modarres, 2010). Traction caused by bending phenomena occurs at the base of different layers of the surface, (Kavussi \& Modarres, 2010). The induced stresses do not result in an immediate failure, but their repetition over time is at the origin of cracking by fatigue, (Di Benedetto et al., 2004). A program of fatigue tests was carried out to determine the resistance to fatigue of recycled asphalt formulated with binders of various degrees of penetrability. The asphalt aggregates comprised three percentages (15\%, $45 \%$ and $75 \%)$ and the temperature of manufacturing included different temperatures. The fatigue test which we conducted involved samples of trapezoidal shape, being tested by bending at two points, (Soltani \& Anderson, 2005). Under review was the maximum deformation experienced by the extreme fiber of the test piece during a sinusoidal amplitude constant arrow application. This deformation was calculated from the arrow head, assuming linear and homogeneous material, (Colbert \& You, 2012). The fatigue in the laboratory test was to determine the deformation $\varepsilon 6$ leading to the rupture of a specimen by fatigue under certain test conditions, (Guthrie et al., 2007).

\section{Parameters of the study}

Three parameters were controlled in this study in order to determine their influence on the resistance to fatigue of the asphalt recycled.

\subsection{Percentage of asphalt aggregate}

Asphalt aggregate percentage is chosen to cover a range from the case of recycling at low rates (15\%), achievable common with most existing asphalt central, until the case of recycling at high rate $(75 \%)$ corresponding to the maximum capacity of recycling in the asphalt central with double drums dedicated to the production of asphalt recycled at high rate. The choice of these two values, maximum and minimum, requires an intermediate point to $45 \%$ of Asphalt aggregate corresponding to the rate of recycling in the asphalt central with deported burner.

\subsection{Manufacturing temperature}

The minimum temperature of manufacturing is chosen equal to $120^{\circ} \mathrm{C}$, this is the temperature to which the old binders start to be remobilized. Used maximum temperature is fixed at $160^{\circ} \mathrm{C}$, or above this temperature, it is in contradiction with the concept of sustainable development that requires a minimum consumption of non-renewable energy. The choice of these two values, maximum and minimum, requires an intermediate point to $140^{\circ} \mathrm{C}$ close to temperature of manufacturing of standard asphalt.

\subsection{Binder penetrability}

The penetrability is the measure of the pressing of a needle normalized in a binder maintained at a fixed temperature, after 5 seconds under a load of $100 \mathrm{~g}$ (Abdelhak et al., 2016-b). In this study we used three binders have got different penetrability ( $42 \mathrm{~m} . \mathrm{m}, 65 \mathrm{~m} . \mathrm{m}$ and $90 \mathrm{~m} . \mathrm{m}$ ) to determine the influence of the latter with the old binder of the asphalt aggregates on the production of a contribution binder compatible with the recycled asphalt that we want to make it.

\section{Materials used}

\subsection{Choices of new aggregates}

New Aggregates choice must be done carefully because its characteristics play a very important role in the quality of the asphalt (Lin et al., 2011). Used aggregates in this study are the fractions $0 / 3,3 / 8$ and $8 / 14$. The (figure 1 ) presents the results of the Particle size analysis test. 


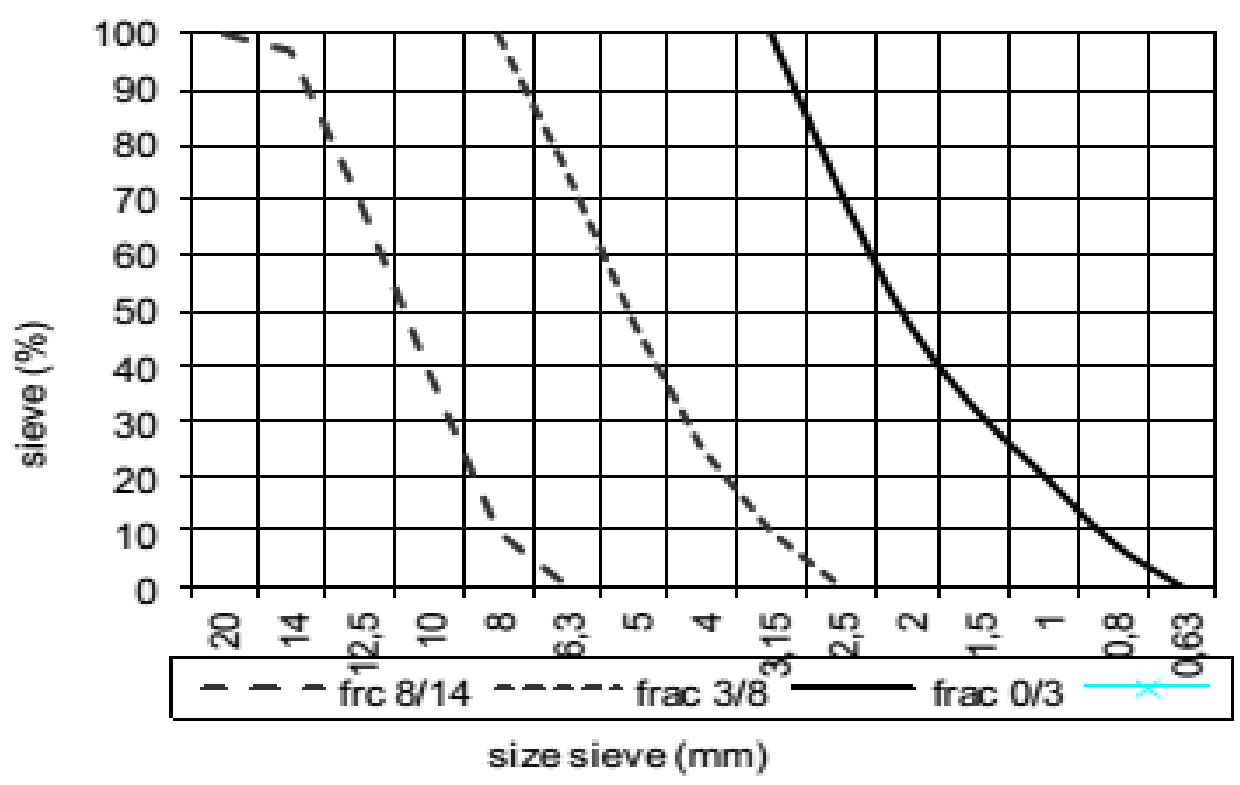

Fig. 1. Particle size analysis of fractions $8 / 14,3 / 8$ and $0 / 3$

The used reagent monomers in STAGAA synthesis process were of two kinds: acrylamide (AM) that provides with nonionic amide group and 2-acrylamido-2-methylpropane sulfonic acid (AMPS) that provides with anionic sulfonic group. Ammonium persulfate (APS) and N,Nmethylenebisacrylamide (NMBA) were used as an initiator agent and a crosslinking agent, respectively.

\subsection{New binders}

The characterization of the bituminous binder mainly reflects the consistency at ambient temperature. This information enables to classify the bituminous binders in rank. Binders used in our study are 30-50, 50-65 and 65-100 classes. There characteristics are presented to the Table 1.

Table 1. Characterization of the new binders.

\begin{tabular}{|c|c|c|c|c|}
\hline Sample & $\begin{array}{c}\text { Penetrability at } 25^{\circ} \mathrm{C}, \\
(1 / 10 \mathrm{~mm})\end{array}$ & $\begin{array}{c}\text { penetrability at } 25^{\circ} \mathrm{C}, \\
(1 / 10 \mathrm{~mm})\end{array}$ & moyen & Specifications \\
\hline \hline Sam 01 & 43 & 40 & 41.5 & $35-50$ \\
\hline Sam 02 & 64 & 64 & 64 & $50-65$ \\
\hline Sam 03 & 92 & 88 & 90 & $65-100$ \\
\hline
\end{tabular}

\subsection{Asphalt Aggregate}

In this study asphalts aggregates used have an age of five years of utilization as layer of bearing. They are shown that recycled asphalt can reach $100 \%$ of asphalt aggregate if it is well calibrated (Lin et al., 2011). For this reason that the asphalts aggregates used were spent in the sieve 14 $\mathrm{mm}$ to eliminate the upper elements to14 $\mathrm{mm}$. After the selection of the elements less than 14 $\mathrm{mm}$ a particle size analysis was conducted as show on the Figure 2. 


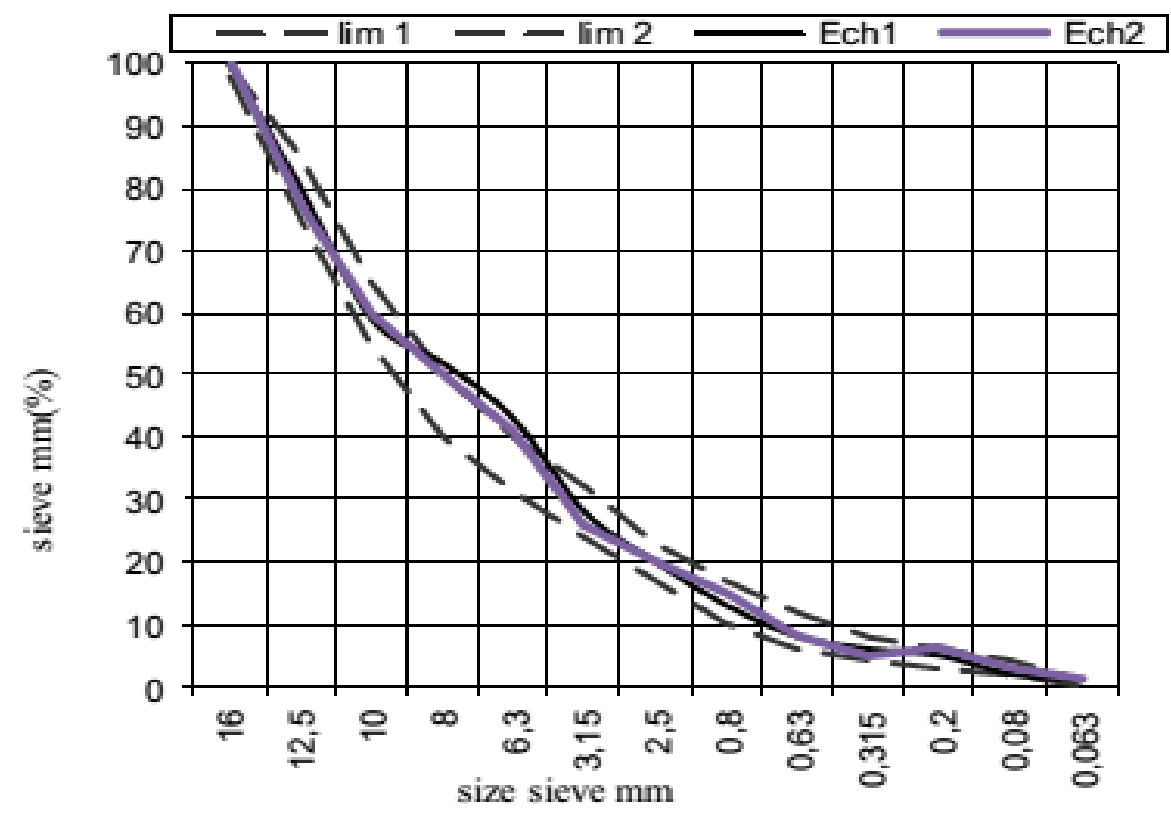

Fig. 2. Particle size analysis of asphalt aggregate

\section{Testing campaign}

\subsection{Formulation of Healthy asphalt}

The Healthy asphalt made is a BBSG (Semi-dense bituminous concrete). The choice of formula through the determination of a mixture having the best ability to compaction and which could give greater stability to the hydrocarbon mixture. Marshal test results in the table. 2 confirmed the percentages of fractions as well as the rate of the binder. The binder used in this formulation is 35-50 class, because this binder is recommended for the BBSG.

This formula of healthy asphalt has been the subject of control by the fatigue test in order to determine its resistance to deformation. The results of the stress test showed that after 15000 cycles the healthy asphalt deforms fast and it begins to take the plastic State. Therefore acceptable ultimate deformation equals $100 \varepsilon 6$ as represented on the figure 3. This ultimate deformation will be considered a limitation agreed for the recycled asphalts to they are going to be studied.

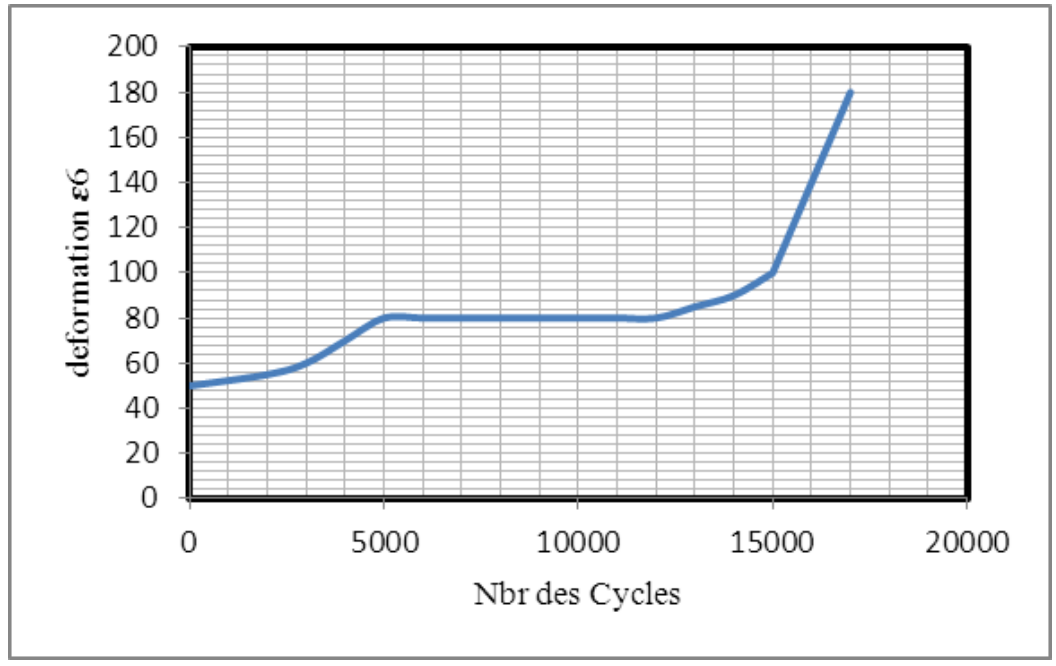

Fig. 3. Fatigue test result on reference healthy asphalt 
Table 2. Results of the Formulation of Healthy asphalt

\begin{tabular}{|c|c|c|}
\hline Frac 8/14 & $45 \%$ & Norme \\
\hline Frac 3/8 & $30 \%$ & \\
\hline Frac 0/3 & $25 \%$ & \\
\hline TL & $5.4 \%$ & $\approx 5.4 \%$ \\
\hline Fluage marshal (F) & 2.60 & F $<4$ \\
\hline Compactness (C) & $96 \%$ & C $>95 \%$ \\
\hline
\end{tabular}

\subsection{Fatigue tests for asphalt recycled}

The table 3 contains formulations that have been programmed in this campaign tests, a control of fatigue as well as the penetrability of the contributions binder have been made for all mixtures of recycled asphalt. Asphalt aggregates dosing Protocol is based on the results of a particle size analysis of each fraction in the healthy mix asphalt, or must cancel the amount of healthy aggregate in each fraction and replace it with the asphalt aggregates. Fatigue test is to apply for the test specimen at a fixed temperature $\left(10^{\circ} \mathrm{C}\right)$ and a fixed frequency $(10 \mathrm{~Hz})$. The fatigue test is also done in mode of deformation. A large number of cycles are applied up to the rupture of the test piece. To test the behavior to fatigue of a mix, at least 4 test tubes must be tested at different levels of deformation (Boudlal et al., 2015).

Table 3. Formulations programmed in this campaign tests

\begin{tabular}{|c|c|c|c|c|c|c|c|}
\hline $\mathrm{N}^{\circ}$ test & $\mathrm{AE} \%$ & $\mathrm{~T}^{\circ}$ & Class of bitume & $\mathrm{N}^{\circ}$ test & $\mathrm{AE} \%$ & $\mathrm{~T}^{\circ}$ & Class of bitume \\
\hline 01 & 15 & 120 & $35-45$ & 15 & 75 & 140 & $50-65$ \\
\hline 02 & 45 & 120 & $35-45$ & 16 & 15 & 140 & $65-100$ \\
\hline 03 & 75 & 120 & $35-45$ & 17 & 45 & 140 & $65-100$ \\
\hline 04 & 15 & 120 & $50-65$ & 18 & 75 & 140 & $65-100$ \\
\hline 05 & 45 & 120 & $50-65$ & 19 & 15 & 160 & $35-45$ \\
\hline 06 & 75 & 120 & $50-65$ & 20 & 45 & 160 & $35-45$ \\
\hline 07 & 15 & 120 & $65-100$ & 21 & 75 & 160 & $35-45$ \\
\hline 08 & 45 & 120 & $65-100$ & 22 & 15 & 160 & $50-65$ \\
\hline 09 & 75 & 120 & $65-100$ & 23 & 45 & 160 & $50-65$ \\
\hline 10 & 15 & 140 & $35-45$ & 24 & 75 & 160 & $50-65$ \\
\hline 11 & 45 & 140 & $35-45$ & 25 & 15 & 160 & $65-100$ \\
\hline 12 & 75 & 140 & $35-45$ & 26 & 45 & 160 & $65-100$ \\
\hline 13 & 15 & 140 & $50-65$ & 27 & 75 & 160 & $65-100$ \\
\hline 14 & 45 & 140 & $50-65$ & & & & \\
\hline
\end{tabular}

\section{Result and Discussion}

From the results of this study, we can distinguish clearly the influence of the rate of aggregate asphalt, the temperature of the manufacturing and class of contribution binder (penetrability) on the resistance of asphalt recycled at the fatigue. For mixtures made with new binder class (35-50) and a temperature of manufacturing equal $\mathrm{T}^{\circ}=120^{\circ}$, resistance to fatigue, represented on the (figure 4), is low for mixes when asphalt aggregate rates of exceeds $25 \%$, reflecting that the temperature was unable to transform the old binder to the presence of the new binder a contribution binder that has the characteristics required by regulation. The (figure 5) represents the results mixtures formulated in new binding class (50-65), when fatigue resistance is acceptable up to a rate of less than $50 \%$ of asphalt aggregate in temperature of the manufacturing equal $120^{\circ}$, this explains the class of the binding agent used to play a role in the cohesion of the components of the mixture and the increase in its resistance to fatigue. However for mixtures when used with new binder is class (65-100) and temperature of the manufacturing $120^{\circ}$ as shown on the (figure 6), fatigue resistance is totally weak because of the contribution binder which is not compatible with the recycled asphalt manufactured BBSG and resistance to the fatigue desired because with a contribution Binder of high penetrability 
cohesion becomes weak. Mixtures made in a temperature of manufacturing equal $\mathrm{T}^{\circ}=140^{\circ}$ or $\mathrm{T}^{\circ}=160^{\circ}$ gave the results have deferential behavior with mixtures produced in a temperature $\mathrm{T}^{\circ}=120^{\circ}$. For an recycled asphalt manufacturing with binder class bitumen (35-50), fatigue resistance is undesirable when the asphalt aggregates rate exceeds $50 \%$, see the (figure 6), this explains the rate of the old binder that influence the new binder to produce a contribution binder of very low penetrability and asphalt it becomes very stiff which causes a fast break. For mixtures made with a binder of class (50-65) the results gave acceptable resistance to fatigue for all formulas because the contribution binder reproduced by new binder and old Binder has got a penetrability compatible with the recycled asphalt formulated (BBSG), presented in the (figure5). However for mixtures made with binder class (65-100), fatigue resistance becomes acceptable to mix them the rate of asphalt aggregate over 50\%, this translated for a low rate asphalt aggregate contribution binder reproduced has very high penetrability, which causes a high flexibility and asphalt recycled it becomes fragile, but with a high rate of asphalt aggregates the old binder without the penetrability of the new binder to produce a contribution binder of desirable penetrability for the manufacturing of recycled asphalt BBSG type in the (figure 5).

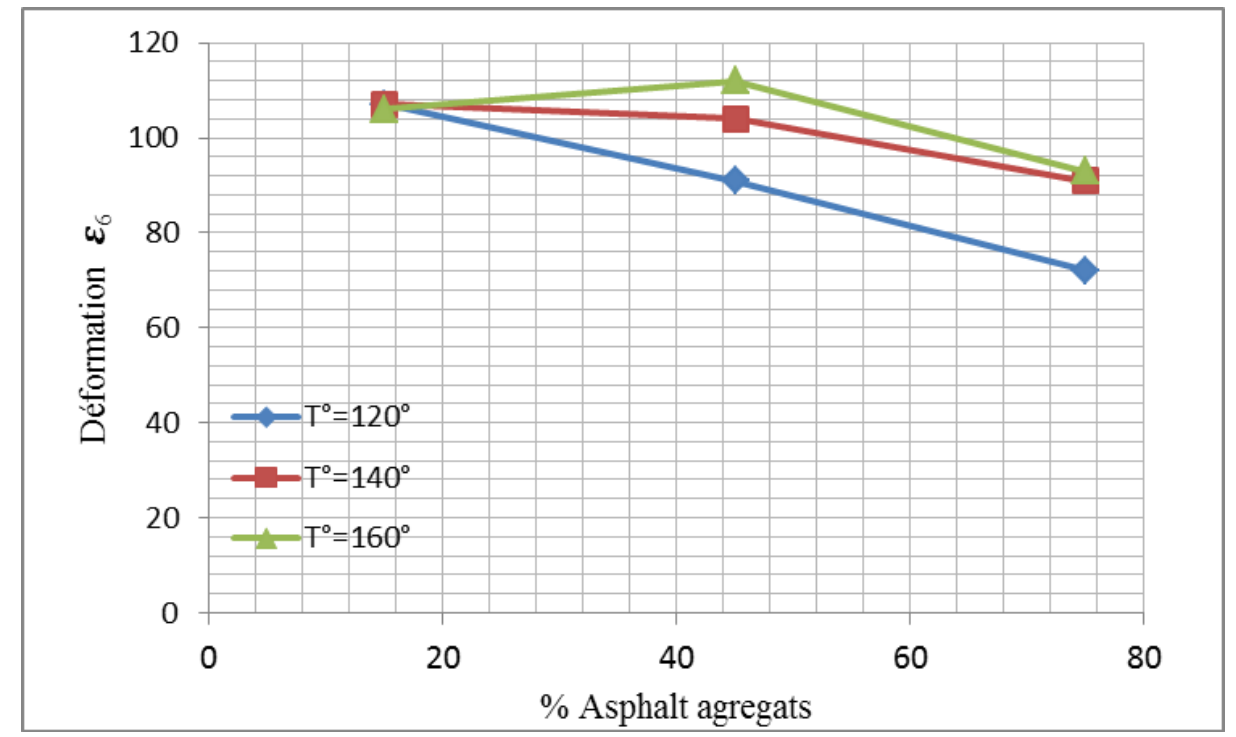

Fig. 4. Effect of the manufacturing temperature and the percentage of asphalt aggregates on the fatigue strength of recycled asphalt manufactured with new binder of penetrability $42 \mathrm{~m} . \mathrm{m}$

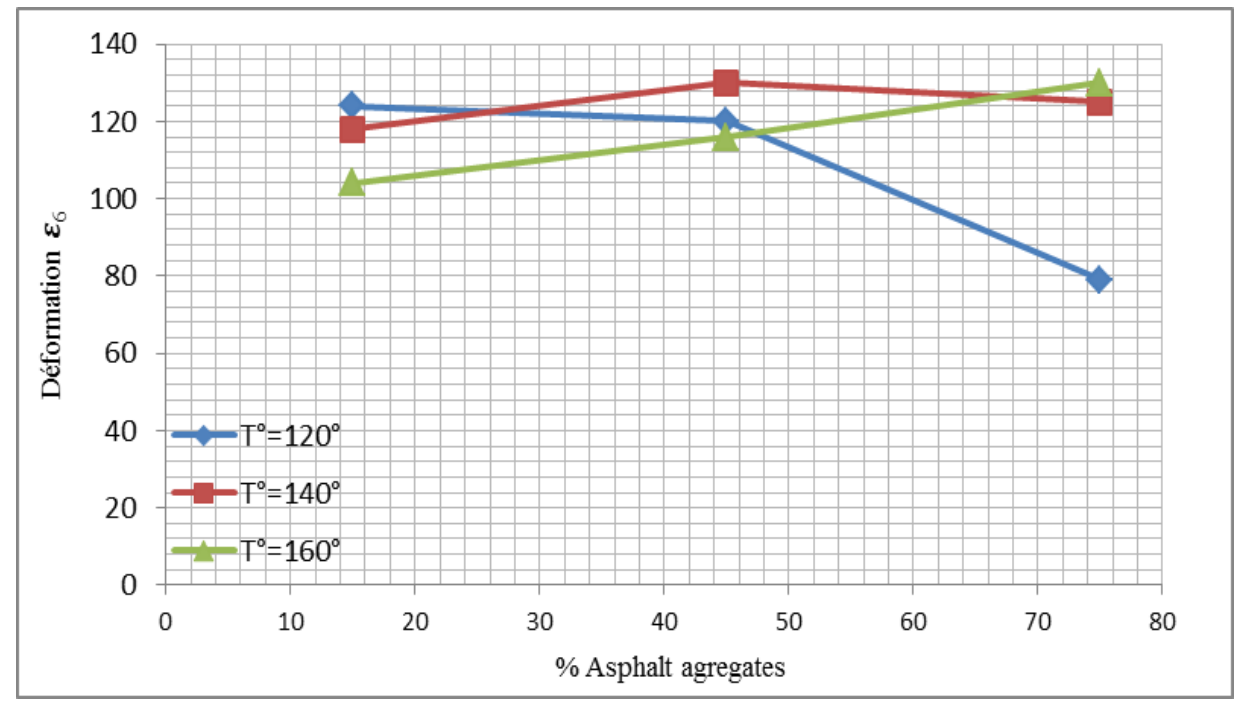

Fig. 5. Effect of the manufacturing temperature and the percentage of asphalt aggregates on the fatigue strength of recycled asphalt manufactured with new binder of penetrability $65 \mathrm{~m} . \mathrm{m}$ 


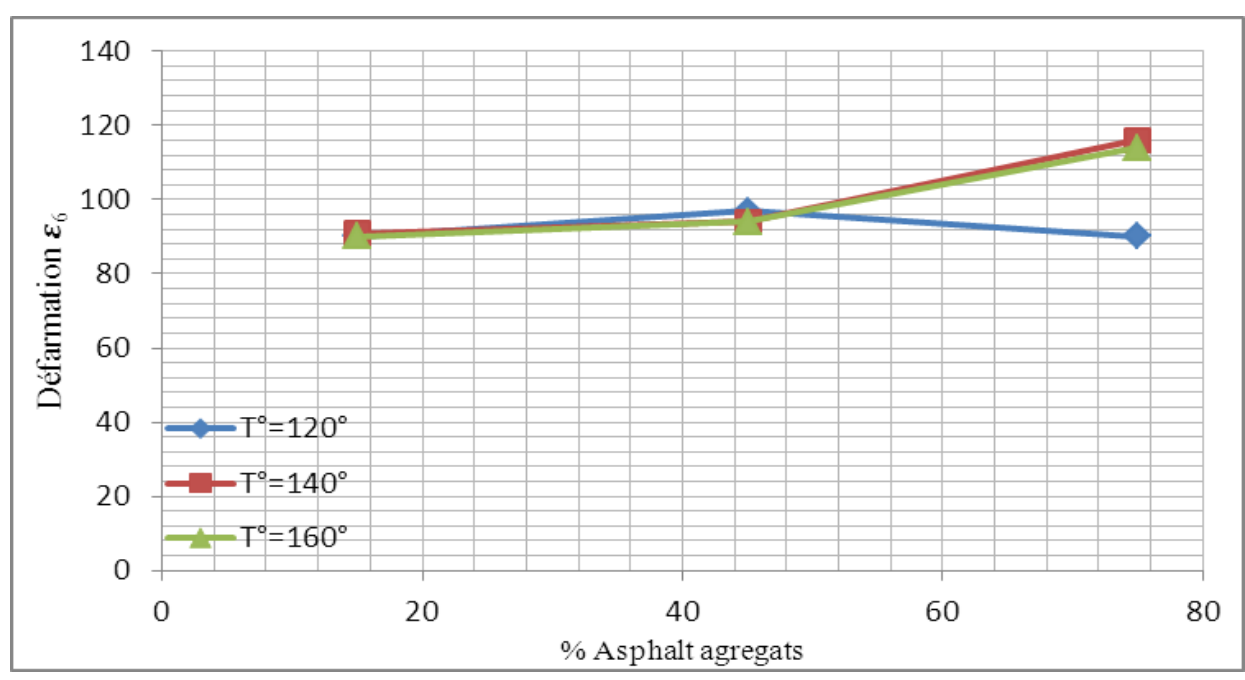

Fig. 6. Effect of the manufacturing temperature and the percentage of asphalt aggregates on the fatigue strength of recycled asphalt manufactured with new binder of penetrability $90 \mathrm{~m} . \mathrm{m}$

\subsection{Optimization of a formulation of recycled asphalt resistant to the rutting}

Following the results presented above, the tests performed by the classic method do not have the capacity to provide accurate results on the rate of AE and the degree of the manufacturing temperature and the class of the binder in the mixture as a function of the mechanical quality desired (fatigue), which has guided the study to the method of the plans of experience (ANOVA). In this experimental plan three factors have been proposed, the answer find is the behavior of the recycled asphalt to fatigue and the influence of factors study on the response for each formula also the compatibility of binders with the performance wish. After operating the factorial experiment that we have chosen as a model of quality, the use of software Minitab.17 We helped to program the necessary testing and the resolution of equations polynomial. The results obtained are the determination of the unknowns of the polynomial presented above in the equation (Eq.1) which expresses the resistance of recycled asphalt to fatigue (response) in function of the parameters of the experience plan (factors). This equation will allow us to determine the resistance to fatigue in function of these variables (factors). Also this equation is a prior justification for the manufacturers of asphalt in the central of production of asphalt.

Fat $=-453,9-3,021 \mathrm{AE}+6,049 \mathrm{~T}^{\circ}+6,584$ Pen $-0,00884 \mathrm{AE}^{2}-0,02052 \mathrm{~T}^{\mathrm{o}}$

- 0,03595 $\mathrm{Pen}^{2}+0,02167 \mathrm{AE}^{*} \mathrm{~T}^{\circ}+0,01250 \mathrm{AE}^{*}$ Pen - 0,01719 T*Pen

According to the results presented on the graph of PARITO in the figure.7, one finds that the interaction between the aggregates of asphalt and the penetrability of the binder plays a crucial role. The interaction between the three factors and the interaction between the temperature of the manufacturing process as well as the penetrability of the binder have shown their influence on the quality of recycled asphalt. These results have confirmed the classic tests performed or we have seen that the rate of aggregate of asphalt contains a percentage of the old binder which affects the mechanical performance of recycled asphalt also the temperature it has a direct relationship with the remobilization of old binder for the put him compatible with the mixture. 


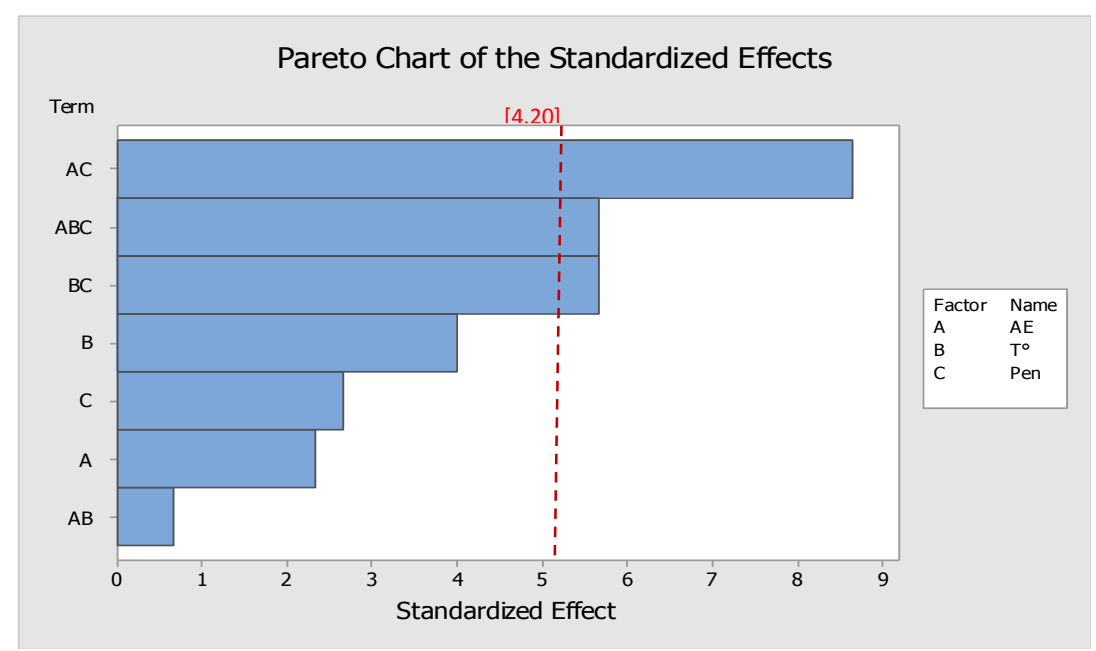

Fig. 7. The Pareto chart of the standardized effects

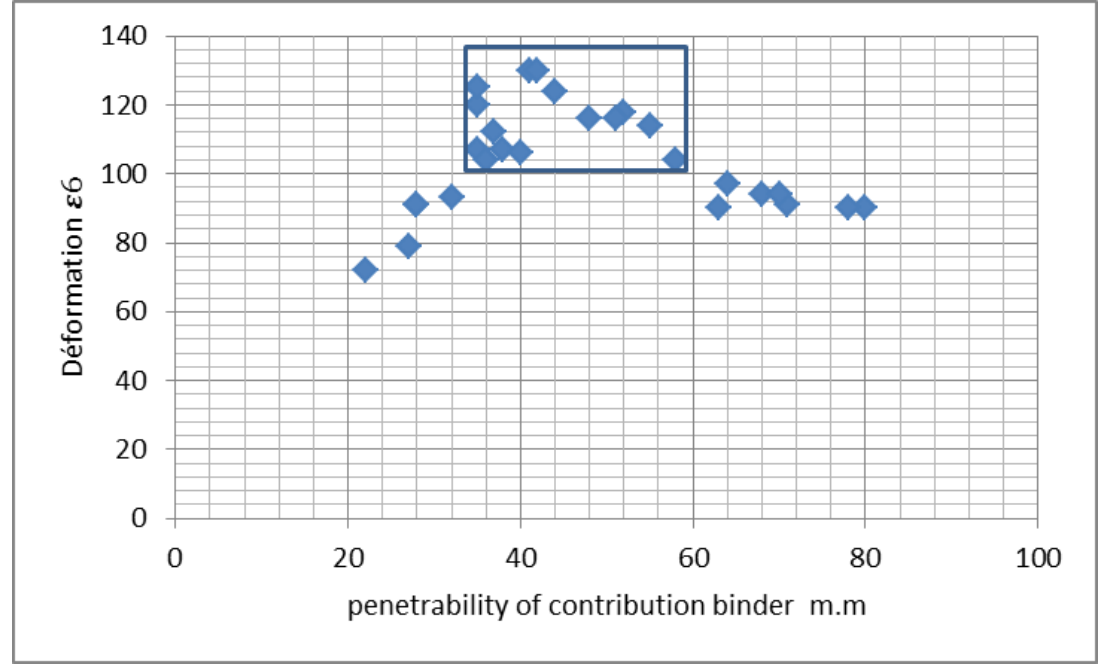

Fig. 8. The desirable domain of the penetrability of contribution binder for manufacturing recycled asphalt resist to the fatigue

\section{Conclusion}

The mechanical behavior of recycled asphalt, in the areas of fatigue, has been studied in this work, and we conclude the following:

1. A rate of $100 \%$ of asphalt aggregate is possible if we can master the choice of a new binder which reproduces a contribution binder compatible with this maximum rate.

2. The temperature must be greater than $140^{\circ}$ to ensure remobilization of the old binder and ensure the homogeneity of the contribution binder.

3. The penetrability or the class of the binder has shown that it plays a key role in the mechanical performance of recycled asphalt. Figure 8. Shows the field limit of the penetrability of the contribution binder to produce a recycled asphalt resistant to fatigue. This limit is $33 \mathrm{~mm}$ inferior and $58 \mathrm{~mm}$ superior.

4. The choice of binder must be made with caution because it is linked to the rate of asphalt aggregate tablets used. If the higher rate of asphalt aggregates is low, the penetrability of new bitumen used must be low, and the contrary for the high rates, in order to produce a contribution binder compatible with the percentage of used asphalt aggregates. 
5. The modeling by the use of the methods of the plans of experience allows you to determine the equation (Eq.6.1) by which can estimate the fatigue for different rates of the factors studied.

6. This study we allow to control the preparation of the binder of intake as amended (old binder+ new binder) with the goal of having the same characteristics of the binder of recycled asphalt. This facilitates the manufacture of recycled asphalt with a high rate of aggregates of asphalt in central.

7. At low temperature the bitumen has a brittle behavior.

8. We can avoid the low-temperature cracking if one uses bitumen of high rank, that is to say, bitumen less "hard" and therefore less "brittle" at low temperature.

9. The bitumen ideal must therefore be both the less likely possible to the phenomena of the fatigue.

\section{References}

Abdelhak, B., Abdelmadjid, H. C., Hamza, G., \& Mohamed, G. (2016-b). Influence of recycled aggregates on the resistance of bituminous concrete in the presence of additives. Revista Romana De MaterialeRomanian Journal of Materials, 46(1), 89-94.

Abdelhak, B., Abdelmadjid, H. C., Mohamed, G., \& Hamza, G. (2016-a). Effect of recycled asphalt aggregates on the rutting of bituminous concrete in the presence of additive. Arabian Journal for Science and Engineering, 41(10), 4139-4145..

Boudlal, O., Khattaoui, M., Djemai, M., \& Medani, M. (2015). Etude Du Comportement Mécanique D'un Mélange De Granulats Naturels Et Du Verre Pour Une Utilisation Dans La Construction Routière (S25). In Congrès français de mécanique. AFM, Association Française de Mécanique.

Colbert, B., \& You, Z. (2012). The properties of asphalt binder blended with variable quantities of recycled asphalt using short term and long term aging simulations. Construction and Building Materials, 26(1), 552-557.

Di Benedetto, H., De La Roche, C., Baaj, H., Pronk, A., \& Lundström, R. (2004). Fatigue of bituminous mixtures. Materials and structures, 37(3), 202-216.

Guthrie, W., Cooley, D., \& Eggett, D. (2007). Effects of reclaimed asphalt pavement on mechanical properties of base materials. Transportation Research Record: Journal of the Transportation Research Board, (2005), 44-52.

Haddad, K., Haddad, O., Aggoun, S., \& Kaci, S. (2017). Correlation between the porosity and ultrasonic pulse velocity of recycled aggregate concrete at different saturation levels. Canadian Journal of Civil Engineering, 44(11), 911-917.

Kalantar, Z. N., Karim, M. R., \& Mahrez, A. (2012). A review of using waste and virgin polymer in pavement. Construction and Building Materials, 33, 55-62.

Kavussi, A., \& Modarres, A. (2010). Laboratory fatigue models for recycled mixes with bitumen emulsion and cement. Construction and Building Materials, 24(10), 1920-1927.

Lin, P. S., Wu, T. L., Chang, C. W., \& Chou, B. Y. (2011). Effects of recycling agents on aged asphalt binders and reclaimed asphalt concrete. Materials and structures, 44(5), 911-921.

Pascal R., (2017). National Project MURE Day of exchange «Contribution of regenerants in the recycling of asphalt mixtures» Reminder of the European and French specifications and prescriptions on the use of (AE) Aggregates of asphalt 15.

Soltani, A., \& Anderson, D. A. (2005). New test protocol to measure fatigue damage in asphalt mixtures. Road materials and pavement design, 6(4), 485-514.

Technical Guides and General Studies Developed by the CTTP (2015). Ministry of Public Works Algeria. 